\title{
Anestesia fuera de pabellón, es tiempo de proyectarla al futuro en su debido lugar
}

\section{Future projection of anesthesia out of the operating room}

María Mercedes Aguirre C. ${ }^{1}$, Carlos Izquierdo A. ${ }^{2}$

\begin{abstract}
Anesthesiology and surgical technics have shown great development over the last decades. This caused several changes in anesthesiology practice, one of them is the growing need for anesthesia out of the operating room. Till now, we are facing this issue in the same way that we used to do in the operating room. Nevertheless, it is time to think about anesthesia in remote locations as a different entity, which has to be faced in a specific form, being able to describe it and to achieve the best results in a cost-effective approach.
\end{abstract}

\section{RESUMEN}

La anestesiología y las técnicas quirúrgicas han mostrado un gran desarrollo en las últimas décadas. Esto ha causado varios cambios en la práctica de anestesiología, uno de ellos es la creciente necesidad de anestesia fuera del quirófano. Hasta ahora, estamos enfrentando este problema de la misma manera que solíamos hacerlo en la sala de operaciones. Sin embargo, es hora de pensar en la anestesia en lugares remotos como una entidad diferente, que debe afrontarse de forma específica, poder describirla y lograr los mejores resultados en un enfoque rentable.

\section{Key words:}

Anesthesia outside the operating room

\section{Palabras clave:}

Anestesia fuera del quirófano

\footnotetext{
Profesor Asociado. Departamento de Anestesiología Facultad de Medicina, Hospital Clínico Universidad de Chile. Cirujano Dentista. Universidad de los Andes.
}

Fecha de ingreso: 15 de abril de 2019

Fecha de aceptación: 20 de mayo de 2019

\section{ORCID}

https://orcid.org/0000-0001-6703-9493

Correspondencia:

María Mercedes Aguirre C.

Email: mmaguirre@uchile.cl 
$\mathrm{D}$ ebido al desarrollo que han experimentado la anestesiología y las técnicas quirúrgicas en los últimos 20 años, la práctica anestésica enfrenta cambios. Uno de los más llamativos es la demanda de soporte anestésico para procedimientos fuera de pabellón, tanto para aquellos diagnósticos como terapéuticos.

Esto obedece a razones de diversa índole. En primer lugar, existe una creciente tendencia a desarrollar técnicas quirúrgicas menos invasivas como la laparoscopía y la cirugía robótica. La introducción de endoscopios cada vez más pequeños, y la introducción de dispositivos cardiacos y endovasculares han contribuido al incremento de la anestesia fuera de pabellón[1].

En segundo lugar, la población está envejeciendo progresivamente. Entre los años 2015 y 2030 el número de personas mayores de 60 años se proyecta que crecerá un 56\%, aumentando de 901 millones a 1,4 billones. La proyección de este número para el 2050 es de 2,1 billones. Por su parte, la población mayor de 80 años está aumentando aún más rápido, proyectándose un aumento desde 125 millones a 434 millones para el 2050. Esto necesariamente nos lleva a presumir que cada vez los pacientes serán más viejos $y$, probablemente, más enfermos[2],[3],[4].

Sin duda, este grupo de pacientes se beneficia de alternativas mínimamente invasivas para resolver sus problemas clínicos.

Por otro lado, el avance tecnológico ha permitido desarrollar técnicas que permiten tratamientos paliativos que mejoran la calidad de vida y la sobrevida de pacientes terminales. También se ha avanzado en la introducción de diversos programas de detección precoz de cáncer y resección endoscópica de lesiones malignas u obtención de biopsias, fundamentalmente digestivas y broncopulmonares[5],[6].

Por último y no menos importante, las políticas de contención de costos y búsqueda de la mayor eficiencia en la productividad clínica se suman a las razones anteriormente expuestas, impulsando en forma permanente la instalación de la anestesia fuera de pabeIlón, contribuyendo a la generación de un listado de procedimientos de diversas especialidades que será cada vez más abultado[3].

¿Qué se espera de los anestesiólogos frente a esta demanda? Verdaderamente nada muy novedoso, ya que estos procedimientos por muy nuevos 0 numerosos que resulten, requieren en mayor o menor medida de analgesia, inmovilidad, amnesia y por supuesto, seguridad garantizada. Esto no constituye mayor desafío para nuestra especialidad, ya que las herramientas técnicas para el logro de estos objetivos están fuertemente consolidadas y somos altamente competentes en su manejo.

La gran dificultad radica en que debemos resolver la anestesia fuera de pabellón de manera eficiente y es con esta eficiencia con la que estamos en deuda. Podemos reconocer algunas razones que explican el por qué no podemos responder en forma eficiente a este contexto, a diferencia de lo que logramos en el pabellón quirúrgico.

En primer lugar, el número y tipo creciente de procedimientos nos obliga a enfrentar escenarios nuevos y desconocidos. Como son nuevos, no hay protocolos de aproximación que contribuyan a obtener buenos resultados en forma sistemática. Por otro lado, como ya hemos dicho, la asociación de pacientes cada vez más viejos y enfermos, con una creciente demanda de atención bajo la modalidad ambulatoria, genera situaciones que aumentan el riesgo o complejizan el escenario, como son la oportunidad de la evaluación y optimización de las condiciones preoperatorias, por ejemplo.

A pesar de todo esto, es innegable el llamado a mejorar la eficiencia de los procesos y a desarrollar sistemas costo-efectivos. Si bien hemos avanzado espectacularmente en seguridad y mortalidad anestésica en nuestra zona segura en el pabellón quirúrgico, debemos reproducirlo fuera de éste. Ahora debemos salir de él adaptándonos a las necesidades y agregarle valor a nuestra prestación, es decir, ofrecer el mejor resultado al menor costo[7].

El principal problema que tenemos para resolver esto, es que no sabemos en realidad de qué hablamos cuando hablamos de anestesia fuera de pabellón. Es decir, tenemos el concepto general, conocemos las características que lo definen y las dificultades que estas nos presentan para nuestra práctica habitual. Sin embargo, no existe una caracterización del escenario que permita específicamente desarrollar estrategias y protocolos conducentes a mejorar la eficiencia y aumentar el valor de nuestra prestación. No sabemos cuánta es la real demanda y número de procedimientos, no sabemos con exactitud quiénes son los pacientes, en qué tipo de salas se realizan los procedimientos mayoritariamente. ¿Existen en realidad diferencias sustantivas con el escenario de pabellón quirúrgico que amerite alguna estrategia especial para mejorar la eficiencia y poder dar certeza de seguridad y buenos resultados? Para responder a esta pregunta, debemos ser capaces de caracterizar y comparar realidades en forma objetiva.

La literatura a este respecto es escasa. El trabajo que mejor respuesta nos da a estas interrogantes es el publicado por Nagrebetsky en abril de 2017[8].

El objetivo de éste fue justamente caracterizar la 
anestesia fuera de pabellón y compararla con la actividad en pabellón quirúrgico, en Estados Unidos de Norteamérica. Se obtuvieron los datos a partir del Registro Nacional de Resultados en Anestesia. Este es un registro electrónico voluntario de todas las prestaciones anestésicas de ese país, que capta alrededor de $25 \%$ de todos los casos. Revisaron 26 millones y medio de anestesias entre enero de 2010 y diciembre de 2014. Los casos de anestesia fuera de pabellón se subdividen en procedimientos gastroenterológicos, cardiológicos y radiológicos intervencionales.

Lo primero que mostró este análisis fue que la proporción de casos fuera de pabellón en relación con el total de casos de anestesia aumentó de un $28,3 \%$ a 35,9\% entre 2010 y 2014.

En cuanto a la caracterización de los casos, los pacientes fuera de pabellón resultaron ser 3,5 años mayores que los de pabellón quirúrgico. Los pacientes mayores de 50 años son más numerosos en el grupo de anestesia fuera de pabellón que en el de pabellones quirúrgicos. Al observar qué ocurre con la edad en el tiempo, se aprecia que en ambos escenarios la edad de los pacientes aumentó durante el período observado, pero con mayor pendiente en el escenario fuera de pabellón. Aquí, la edad promedio observada en 2010 fue de 51,5 años y aumentó en 3,5 años al 2014. Los pacientes envejecieron a razón de 0,87 años por año fuera de pabellón y a 0,66 años por año en pabellones quirúrgicos.

En relación con la complejidad de los pacientes, son más numerosos los pacientes ASA III-IV fuera de pabellón que en pabellón quirúrgico. También aumentó la puntuación de ASA durante el intervalo de tiempo observado en ambos grupos, con mayor tendencia en el grupo fuera de pabellón, pero la diferencia no fue clínicamente importante.

En cuanto a la duración de los procedimientos, aquellos resueltos fuera de pabellón demoran en promedio la mitad del tiempo que aquellos realizados en pabellones quirúrgicos.

Un hallazgo llamativo fue que un mayor número de procedimientos fuera de pabellón comienza en horario no hábil, en comparación con los de pabellones quirúrgicos. Esto se puede interpretar pensando que existe un mayor número de casos que son de urgencia y que se resuelven mediante intervenciones fuera de pabellón. Sin embargo, esta no parece ser la realidad puesto que no se observa lo mismo durante los fines de semana. La interpretación es que este fenómeno habla de un sistema menos eficiente y ordenado administrativamente que la programación y cumplimiento de la agenda de los pabellones quirúrgicos.

En cuanto a la modalidad de atención, la propor- ción de pacientes ambulatorios aumentó en los dos escenarios durante el intervalo de tiempo observado, con una mayor tendencia en el grupo fuera de pabellón.

Al caracterizar el tipo de procedimientos que se realizaron fuera de pabellón entre los años 2010 y 2014, se observa que la marcada mayoría de casos ocurren en las salas de procedimientos gastroenterológicos; cerca del $80 \%$. Dentro de estos, el principal procedimiento lo constituyen las colonoscopías (Figura 1).

Estas han aumentado llamativamente en el tiempo, llegando a ser el $50 \%$ del total de las intervenciones fuera de pabellón en el año 2014.

Conocidos algunos elementos que nos ayudan a caracterizar el escenario fuera de pabellón, debemos pensar en cómo garantizar la seguridad del paciente en este contexto. Ciertamente la especialidad ha logrado altos niveles de seguridad con el avance tecnológico incorporado a la monitorización, equipos de administración de anestesia y desarrollo de drogas de mejores perfiles farmacocinéticos y farmacodinámicos. Tanto así que la mortalidad atribuida a la anestesia ha caído de 357 por millón antes de los años 70 a 34 por millón entre los años 90 y 2000[9].

Pero esto es una realidad medida y declarada en nuestras intervenciones asociadas a procedimientos realizados en pabellones quirúrgicos. Seguimos ignorantes respecto a cifras fuera de pabellón. Nuevamente se hace evidente que es necesario caracterizar los riesgos y complicaciones propios y específicos de este escenario, puesto que es diferente.

Lamentablemente, la evidencia disponible para responder esta interrogante es escasa. Sharma publica una incidencia de complicaciones cardiorrespiratorias en pacientes ambulatorios sometidos a endoscopías digestivas de $0,9 \%$. Los factores de riesgo

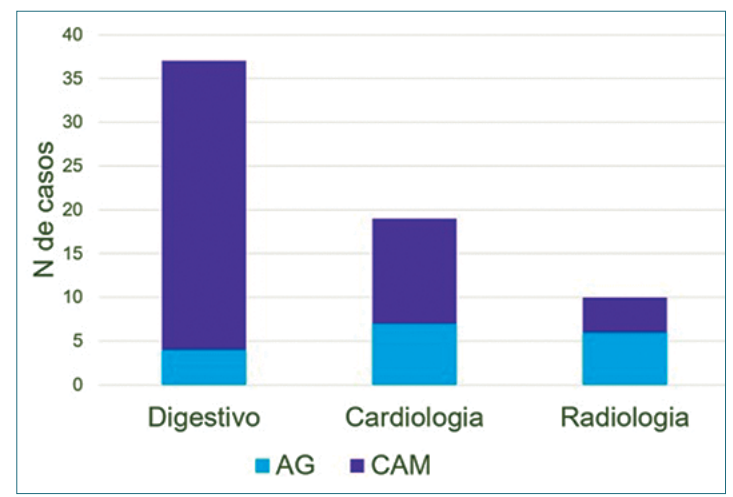

Figura 1. Lugar de ocurrencia y técnica anestésica. 
identificados en esta serie fueron la edad mayor a 60 años, ASA mayor y pacientes hospitalizados versus ambulatorios. La incidencia de hospitalizaciones no planificadas fue de 27/100 mil[10].

En cuanto al eventual rol en la seguridad, que recae en el profesional a cargo de la sedación en este escenario particular (enfermero(a) anestesista, enfermero(a) entrenado(a) en sedación, anestesiólogo, gastroenterólogo), a partir de la revisión de Leslie acerca de complicaciones y hospitalizaciones no planeadas en procedimientos fuera de pabellón, se puede concluir que faltan estudios estandarizados que demuestren que una opción es más segura que otra. Interesante asunto en que la investigación debe ahondar, puesto que a pesar de que teóricamente se tienda a pensar que la alternativa del médico anestesiólogo es en esencia la más segura, tal vez no es así y se abre una puerta para poder dar respuesta a la creciente demanda de sedación para procedimientos endoscópicos, la cual no podrá ser asumida por el recurso anestesiólogo tanto por un problema de oferta de éste, como por la arista económica[10],[11].

Concluye Leslie también que dada la escasa y poco estandarizada literatura al respecto, los riesgos y frecuencia de eventos adversos fuera de pabellón, no están bien caracterizados ni cuantificados, comparado con lo que sabemos que ocurre en pabellón quirúrgico.

Tal vez la mejor mirada de la que disponemos hoy al respecto, nos la entrega Woodward quien junto a sus colaboradores revisaron los casos cerrados por demandas originadas en procedimientos fuera de pabellón, obtenidos a partir de la base de datos de los casos cerrados de anestesia (Anesthesia Closed Claims) ocurridos entre los años 2000 y 2012 y los comparan con las demandas originadas en pabellón quirúrgico[12].

Además, revisaron los resultados de las anestesias fuera de pabellón, usando el registro nacional de outcomes en anestesia y lo compararon con lo hallado en el ASA Closed Claims.

Este registro comprende 22 compañías que aseguran 1/3 de los anestesiólogos en Estados Unidos de Norteamérica. Los investigadores evaluaron la causa de la lesión, la severidad y el resultado final de ésta. Este reporte está realizado sobre 10.357 demandas relacionadas con cirugía o anestesia: 1.900 fueron originadas por la anestesia; 72 fuera de pabellón y 1.828 en pabellón quirúrgico. Recordemos que el Registro Nacional de Resultados Anestésicos (NACOR), es un reporte voluntario y comprende aproximadamente el $25 \%$ de anestesias en Estados Unidos. Entre 2010 y 2013 se registraron 9 millones de procedimientos anestésicos.
En cuanto a las características generales de los eventos, aquellos ocurridos fuera de pabellón involucraron con mayor frecuencia pacientes menores de 16 años y pacientes con ASA mayor o igual a III, en comparación con los eventos ocurridos en pabellones quirúrgicos.

La mayoría de los eventos fuera de pabellón ocurrieron en las salas de procedimientos digestivos ( $51 \%$ ) y el $89 \%$ de éstos, se realizaron bajo la modalidad de Cuidados Anestésicos Monitorizados (CAM). El segundo y tercer lugar lo ocupan las salas de procedimientos intervencionales cardiológicos y radiológicos respectivamente, donde la anestesia general se ve mayormente representada (Figura 1).

En relación con la frecuencia de ocurrencia de eventos que llevaron a las demandas y a la severidad de éstos, es necesario destacar que la proporción de demandas por muerte es el doble fuera de pabellón que en pabellón quirúrgico, y las lesiones temporales no discapacitantes son causa más frecuente de demandas en pabellón quirúrgico (Figura 2).

En cuanto al mecanismo de injuria, la proporción de eventos respiratorios es mayor al doble fuera de pabellón versus pabellón quirúrgico $(53 \%$ versus $23 \%)$, siendo la inadecuada ventilación/oxigenación la principal causa fuera de pabellón y muy superior a la incidencia en pabellón quirúrgico (31\% versus $6 \%)$. Otras causas respiratorias incluyeron aspiración de contenido gástrico (11\%), intubación difícil (7\%) e intubación esofágica (7\%). Causas cardiovasculares, errores en medicación o fallas de equipos no difirieron entre ambos escenarios.

La sobresedación y consecuente depresión respiratoria fue considerada responsable del evento en 20 casos ocurridos fuera de pabellón. Dos tercios de ellos

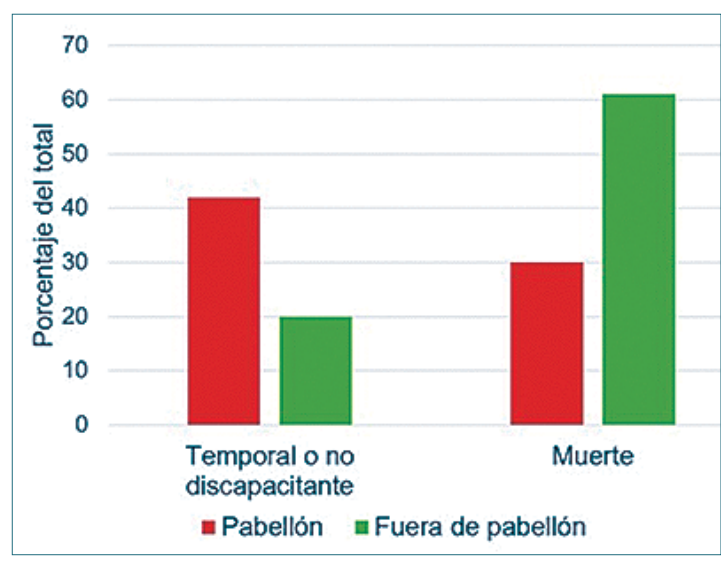

Figura 2. Severidad de las lesiones en casos fuera de pabellón vs pabellón. 
ocurrieron en salas de procedimientos digestivos.

El propofol usado como droga única o en combinación con otras, fue la droga con mayor frecuencia involucrada en la sobresedación, considerándose que en $68 \%$ de los casos, ésta podría haber sido prevenida con mejor monitorización (Figura 3).

El cuidado anestésico se consideró subestándar en el $88 \%$ de los casos. Todos los casos de sobresedación resultaron en muerte o daño cerebral permanente.

A modo de resumen, fuera de pabellón los CAM son la técnica anestésica que mayor número de eventos que llevan a demandas produce, en comparación con aquellos originados en pabellón quirúrgico. Los eventos respiratorios constituyen el evento crítico más frecuente fuera de pabellón y la cifra es superior a lo ocurrido en pabellón quirúrgico. En mucho mayor porcentaje el evento se considera prevenible con mejor monitorización fuera de pabellón que en pabellón quirúrgico (Figura 4).

Tomando esta información, que si bien tiene un sesgo, pues no representa el total del universo de los procedimientos fuera de pabellón, sino sólo caracteriza a aquellos en los cuales se produjo un evento crítico que llevó a litigio y fallo, igualmente resulta valiosa por ser de la escasa disponible y por lo gravitante de su contenido, podemos concluir lo siguiente. Si la obligada interrelación entre sobresedación, inadecuada ventilación son la gran causa de los principales eventos críticos graves que se están produciendo fuera de pabellón, con mayor frecuencia que en pabellón quirúrgico, resulta evidente que la incorporación del capnógrafo como monitor obligado de la ventilación, debe ser un estándar en todo escenario donde se desarrolle la anestesia fuera de pabellón. Y tal es así que está ya recomendado en numerosas guías clínicas al respecto[13],[14],[15],[16].

Para finalizar, luego de este análisis acerca de la dimensión actual de la anestesia fuera de pabellón, podemos hacer las siguientes reflexiones con un espíritu crítico y una mirada de proyección. Debemos llevar el capnógrafo fuera de pabellón, sin excepciones. Debemos describir la realidad local, sólo de esa manera podremos identificar nuestros riesgos y podremos generar estrategias para mejorar la seguridad y calidad de atención. Debemos trabajar y estar atentos al desarrollo de nuevas drogas y sistemas de administración, cuyos perfiles farmacocinéticos y farmacodinámicos se ajusten mejor al modelo de las necesidades clínicas que nos exige este escenario; procedimientos cortos y ambulatorios la gran mayoría, con escaso dolor postprocedimiento. Por ejemplo, está en fase de desarrollo un agonista GABA Remimazolam: molécu-

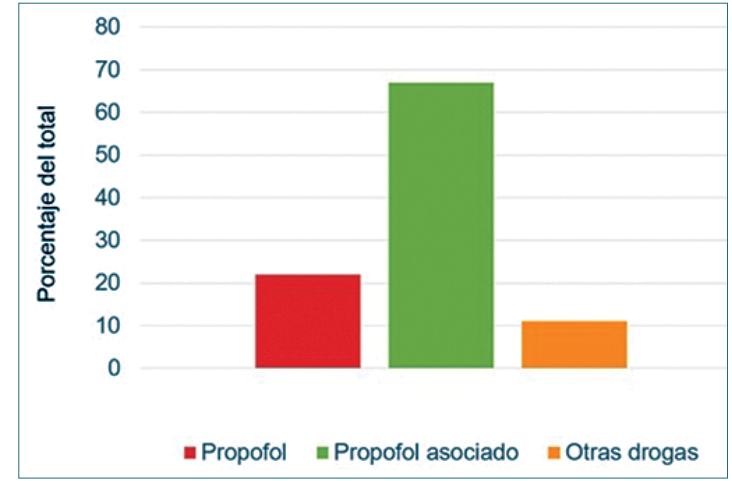

Figura 3. Agentes involucrados.

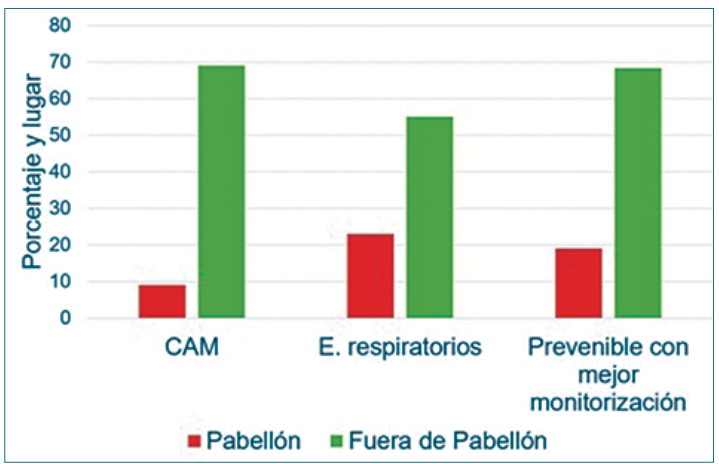

Figura 4. Características demandas pabellón vs fuera de pabellón.

la con enlaces ester inestables, que se metaboliza por esterasas plasmáticas no específicas. Posee un inicio de acción más rápido y una recuperación 4 veces más rápida que con midazolam. Se revierte con flumazenil en 60 segundos y produce menos depresión respiratoria.

Existe otro agonista GABA no benzodiacepínico, $J M-1232$. Posee un inicio y término de acción más breve que midazolam. Existe cierta evidencia de que tiene cierto efecto antinociceptivo, por lo que evitaría el uso de opioides.

Se están investigando también análogos de etomidato que respondan a este perfil específico deseado en sedaciones de corta duración (ABP-700 o MOCMetomidato) y que carezcan del efecto de supresión adrenal (MOC-etomidato).

Por su parte, están en investigación opioides moduladores de receptores mu, que produce un efecto selectivo analgésico, pero no los efectos colaterales, fundamentalmente, la depresión respiratoria (PMZ21 y TRV130). Cebranopadol, otro fármaco que ya ha 
iniciado la etapa de estudios clínicos, tendría mejor analgesia con menor desarrollo de tolerancia.

También se encuentran en desarrollo derivados de propofol y ketamina, buscando que posean un rápido inicio y fin de efecto clínico, con menos efectos adversos[1].

También se trabaja en la generación de sistemas de administración que respondan a la necesidad clínica específica en forma más reproducible y segura, como los sistemas de asa cerrada.

Es fundamental avocarse a implementar gestión de recursos en forma más eficiente. Mejorar modelos clínicos administrativos, como los sistemas de agendamiento y la oportunidad de evaluación de los pacientes previo a los procedimientos, evitando suspensiones. Gestionar en forma eficiente el personal y las salas de trabajo. Por ejemplo, tender a la construcción de áreas centralizadas donde se realicen diversos tipos de procedimientos, en lugar de que estos se repartan por diferentes y lejanas dependencias de una institución, con las consecuentes dificultades que esto acarrea. Así mismo, las nuevas construcciones o remodelaciones de instalaciones deben contemplar espacios para la instalación segura y funcional de la estación de anestesia y su personal. Se debe mantener el énfasis en promover la permanente comunicación entre los equipos.

Es importante darle un espacio de mayor preponderancia en el currículo de la formación de anestesiólogos a la anestesia fuera de pabellón, de manera que no sea sólo una extrapolación de lo que aprenden en pabellón quirúrgico. Debemos hacernos cargo de educar al personal no anestésico acerca del riesgo de la sedación y de sus requerimientos.

Finalmente, debemos tomar conciencia de que el anestesiólogo no estará más confinado solo al pabellón quirúrgico y dejar de tratar de enfrentar la anestesia fuera de pabellón como una simple extrapolación de lo que hacemos en pabellones quirúrgicos. Hay que entender que este es un modelo diferente, que tiene características especiales, que, aunque hasta aquí, hemos sabido dar respuesta, no logramos el calce perfecto. Los tiempos y la realidad cambian y estamos llamados a hacer frente a la alta demanda de anestesiólogos y/o prestaciones anestésicas en distintos frentes, en forma eficiente y segura, tal como llevamos años haciéndolo en el pabellón quirúrgico.

\section{Referencias}

1. Boggs SD, Barnett SR, Urman $\mathrm{RD}$. The future of nonoperating room anesthesia in the $21 \mathrm{st}$ century: emphasis on quality and safety. Curr Opin Anaesthesiol. diciembre de 2017;30(6):644-51.

2. United Nations, Department of Economic and Social Affairs, Population Division (2015). World Population Ageing 2015 (ST/ESA/ SER.A/390).

3. Eichhorn V, Henzler D, Murphy MF. Standardizing care and monitoring for anesthesia or procedural sedation delivered outside the operating room: Curr Opin Anaesthesiol. agosto de 2010;23(4):494-9.

4. Chang B, Urman RD. Nonoperating Room Anesthesia. Anesthesiol Clin. marzo de 2016;34(1):223-40. https:// doi.org/10.1016/j.an- clin.2015.10.017.

5. Dabu-Bondoc S. NonOperating Room Anesthesia: distancing from invasive surgery, embracing the era of interventional medicine. Curr Opin Anaesthesiol. diciembre de 2017;30(6):63943. https://doi.org/10.1097/ AC0.0000000000000524.

6. Voulgarelis S, Scott JP. Monitoring for Nonoperating Room Anesthesia. Anesthesiol Clin. diciembre de 2017;35(4):591-9. https://doi.org/10.1016/j.anclin.2017.07.004.

7. Recart F A. Medicina perioperatoria ¿El futuro de la anestesiología? Rev Chil Anest. 7 de septiembre de 2018;47(3):166-165.

8. Nagrebetsky A, Gabriel RA, Dutton RP, Urman RD. Growth of Nonoperating Room Anesthesia Care in the United States: A Contemporary Trends Analysis. Anesth Analg. abril de
2017;124(4):1261-7.

9. Bainbridge D, Martin J, Arango M, Cheng D. Perioperative and anaesthetic-related mortality in developed and developing countries: a systematic review and meta-analysis. The Lancet. septiembre de 2012;380(9847):1075-81. https://doi.org/10.1016/S01406736(12)60990-8.

10. Sharma VK, Nguyen CC, Crowell MD, Lieberman DA, de Garmo $P$, Fleischer DE. A national study of cardiopulmonary unplanned events after GI endoscopy. Gastrointest Endosc. julio de 2007;66(1):27-34. https://doi. org/10.1016/j.gie.2006.12.040.

11. Leslie K, Kave B. Complications and unplanned admissions in nonoperating room procedures: Curr Opin Anaesthesiol. diciembre de 2017;30(6):658-62.

12. Woodward ZG, Urman RD, 
Domino KB. Safety of NonOperating Room Anesthesia. Anesthesiol Clin. diciembre de 2017;35(4):569-81. https://doi.org/10.1016/j.anclin.2017.07.003.

13. Sociedad Anestesiología de Chile. Anestesia fuera de pabellón recomendaciones. https://www. sachile.cl/upfiles/rc/RC_Anestesia_Fuera_de_Pabellon_SACH. pdf Buscada el 02/04/2019.

14. Checketts MR, Alladi R, Ferguson K, Gemmell L, Handy JM, Klein
AA, et al. Recommendations for standards of monitoring during anaesthesia and recovery 2015: Association of Anaesthetists of Great Britain and Ireland. Anaesthesia. enero de 2016;71(1):85-93.

15. Practice Guidelines for Moderate Procedural Sedation and Analgesia 2018: A Report by the American Society of Anesthesiologists Task Force on Moderate Procedural Sedation and Analgesia, the American Association of
Oral and Maxillofacial Surgeons, American College of Radiology, American Dental Association, American Society of Dentist Anesthesiologists, and Society of Interventional Radiology*. Anesthesiology. marzo de 2018;128(3):437-79.

16. Sedation in under 19s: using sedation for diagnostic and therapeutic procedures. Clinical guideline Published: 15 December 2010 nice.org.uk/guidance/ cg112 Revisadas en 2018. 